\title{
ESTRATÉGIAS ALTERNATIVAS DE IMPLEMENTAÇÃO DO EFEITO PROTETOR CONTRA O DANO MUSCULAR
}

\author{
Livia Elias Delgado, Universidade Estadual Paulista - UNESP, Rio Claro, São Paulo - \\ Brasil
}

Leonardo Coelho Rabelo de Lima, Universidade Estadual Paulista - UNESP, Rio Claro, São Paulo - Brasil

\section{RESUMO}

Já foi bem estabelecido que, após a realização de exercícios aos quais não se está acostumado, principalmente se esses envolverem contrações excêntricas, ocorre um processo conhecido como dano muscular (DM). Esse processo consiste na desorganização e/ou rompimento de células musculares graças a elevados níveis de estresse mecânico e/ou metabólico. Sabe-se que, após a ocorrência do DM, o músculo acometido se recupera e passa a ser mais resistente a esse fenômeno, apresentando respostas atenuadas desses sintomas, fenômeno conhecido como efeito protetor (EP). Entretanto, para a manifestação deste efeito por meio de contrações excêntricas, é necessária a ocorrência de DM de alta magnitude, o que pode não ser interessante para atletas e entusiastas da atividade física. Recentemente, diferentes estratégias de proteção contra o DM que não envolvem a realização de contrações excêntricas máximas vêm sendo apresentadas, como a realização de contrações excêntricas submáximas, contrações isométricas, treinamento de flexibilidade e aumento da temperatura muscular previamente ao exercício. O objetivo do presente estudo foi revisar essas estratégias de proteção contra o DM para melhor entender esse fenômeno. Foi realizado um levantamento em importantes bases de dados e, os artigos encontrados e considerados relevantes ao tema foram revisados e didaticamente explanados ao longo do trabalho.

Palavras-Chave: Dano muscular; Efeito protetor; Flexibilidade; Contrações isométricas; Aquecimento.

\section{ALTERNATIVE STRATEGIES FOR THE IMPLEMENTATION OF THE PROTECTIVE EFFECT AGAINST MUSCLE DAMAGE}

\begin{abstract}
It has been well established that, after performing unaccustomed exercise, especially if it involves eccentric contractions, a process known as muscle damage (MD) occurs. This process consists in the tearing and/or disruption of muscle cells due to high levels of mechanical and metabolic stress. It is known that, after the occurrence of MD, the affected muscle recovers and becomes more resistant to this phenomenon, showing attenuated symptoms, a phenomenon known as protective effect (PE). However, for the manifestation of this effect, eccentric contractions are required, which leads to high magnitudes of MD that may not be interesting for athletes and physical activity enthusiasts. Recently, different protection strategies against MD that does not involve performing maximal eccentric contractions have been presented, such as carrying out submaximal eccentric contractions, isometric contractions, flexibility training and increasing muscle temperature prior to exercise. The aim of this study was to review these strategies of protection against MD, in order to better understand this phenomenon. A survey was conducted in important
\end{abstract}


databases, and papers found and deemed relevant to the topic were reviewed and didactically explained throughout the paper.

Key-Words: Muscle damage; Protective effect; Flexibility; Isometric contractions; Heating.

\title{
ESTRATEGIAS ALTERNATIVAS PARA LA APLICACIÓN DEL EFECTO PROTECTOR CONTRA EL DAÑO MUSCULAR
}

\begin{abstract}
RESUMEN
Ha sido bien establecido que, después de la realización de ejercicios a los que uno no está acostumbrado, sobre todo si éstas implican contracciones excéntricas, un proceso conocido como daño muscular (DM) se produce. Este proceso consiste en la interrupción y la interrupción de las células musculares debido a altos niveles de estrés y/o el metabolismo mecánica. Se sabe que, después de la ocurrencia de la MS, el músculo afectado se recupera y se vuelve más resistente a este fenómeno, que muestra atenuado estos síntomas, un fenómeno conocido como efecto protector (PE) respuestas. Sin embargo, para la manifestación de este efecto por medio de contracciones excéntricas, se requiere la presencia de DM de alta magnitud, que puede no ser interesante para los atletas y los entusiastas de la actividad física. Recientemente, diferentes estrategias de protección contra la DM que no esté relacionado con la realización de contracciones excéntricas máximas se han presentado, como por ejemplo la realización de contracciones excéntricas submáximas, contracciones isométricas, entrenamiento de la flexibilidad y el aumento de la temperatura muscular antes del ejercicio. El objetivo de este estudio fue revisar estas estrategias para proteger contra la DM para comprender mejor este fenómeno. Se realizó una encuesta en las bases de datos y documentos pertinentes encontrado y que se considere relevante para el tema fueron revisados y explicó didácticamente en todo el documento.
\end{abstract}

Palabras-Claves: Daño muscular; Efecto protector; Flexibilidad; Contracciones isométricas; Calentar. 


\section{INTRODUÇÃO}

A força muscular é um tema muito estudado na atualidade, por seu desenvolvimento ser importante para a manutenção da saúde e para o aprimoramento do rendimento de atletas de alto nível. É de suma importância, portanto, entender seus mecanismos para poder melhor usufruir dos seus benefícios.

O desenvolvimento da força envolve adaptações tanto neurais como morfológicas. No inicio de um treinamento temos preferencialmente adaptações neurais ${ }^{1}$ com as quais se melhora a coordenação intermuscular e aumenta-se a ativação do músculo, permitindo que, durante uma contração, mais unidade motoras e, por consequência, mais fibras musculares sejam recrutadas. As adaptações morfológicas passam a aumentar sua contribuição após o período inicial, e envolvem o aumento do tamanho das fibras ou área de secção transversa (hipertrofia) bem como do número das mesmas (hiperplasia).

O músculo realiza três tipos de contração, a concêntrica (CON), isométrica (ISO) e a excêntrica (EXC). Contrações que exigem o encurtamento das fibras musculares, em que a origem de um músculo se aproxima de sua inserção, são caracterizadas como CON. Ao se realizar o afastamento ou alongamento das fibras enquanto tensionado, o músculo se contrai EXC. A contração ISO acontece quando há a sustentação de uma carga, e o músculo permanece em um comprimento fixo, sem movimentação articular. ${ }^{2}$

Há um consenso na literatura apontando que contrações EXC levam a um fenômeno conhecido como dano muscular (DM). ${ }^{3} \mathrm{O}$ DM é caracterizado por microlesões causadas por intensas contrações (de caráter EXC, principalmente) realizadas pelo tecido muscular. ${ }^{4}$ Por impor uma elevada tensão nas fibras musculares, a ação EXC provoca DM em magnitudes elevadas, induzindo diversos processos mecânicos como a desorganização da linha $\mathrm{Z}$ dos sarcômeros, assim como a perda de mitocôndrias e rupturas ou modificações estruturais nos miofilamentos de actina e miosina. ${ }^{2-5}$ As fibras do tipo II são mais recrutadas nas ações EXC e, diferentemente das do tipo I, são mais curtas, sendo mais suscetíveis ao DM.

Alguns dos principais motivos pelos quais se investiga o DM são: para se mensurar o período de recuperação necessário entre as sessões de treinamento, importante para a Conexões: revista da Faculdade de Educação Física da UNICAMP, Campinas, v. 12, n. 3, p. 79-105, jul./set. 2014. 
elaboração de programas de exercícios; e como indicador de treinamentos de alta intensidade, principalmente em estudos sobre o efeito de carga repetida induzida pelo treinamento de força. ${ }^{3}$ Sabe-se também, que, uma vez danificado, o tecido muscular passa por um processo de regeneração que o torna mais resistente contra DM futuro. Esse efeito de resistência contra o DM induzido por uma sessão prévia em que há danificação é denominado de efeito protetor (EP) e pode se manifestar em diversas magnitudes. ${ }^{6}$ Uma série de estudos investigou diferentes fatores que influenciam o EP contra o DM. Nosaka et al., ${ }^{7}$ por exemplo, demonstraram que exercícios EXC realizados com o músculo em um estado alongado provocam DM maior que exercícios feitos com a musculatura menos alongada e, consequentemente, um maior EP. Pesquisas recentes utilizaram outros tipos de contrações com a finalidade de averiguar se as mesmas atenuariam o DM induzido por exercícios EXC posteriores, descobrindo que de fato elas causam um EP, porém, de menor magnitude.

A flexibilidade de um músculo, por exemplo, é um dos fatores que pode atenuar o DM. Dados apresentados por Chen et al. ${ }^{8}$ indicam que o treinamento de flexibilidade pode causar um EP semelhante àquele gerado por contrações EXC contra o DM causado por sessões de exercício EXC. Acredita-se que, devido ao aumento da complacência muscular resultante do treinamento de flexibilidade, há um aumento da proteção contra o DM. ${ }^{9}$ Outra possibilidade é o fato de se aumentar o numero de sarcômeros em série com treinos de flexibilidade. Entretanto mais estudos devem ser realizados para que se possa comprovar essa teoria.

Alternativamente, estudos como os de Chen et al. ${ }^{10}$ e Chen et al. ${ }^{11}$ demonstram que mesmo a realização de contrações ISO antes de contrações EXC, leva a níveis atenuados de DM. Verificou-se que contrações ISO voluntárias máximas induzem um EP mais eficiente, no sentido de utilizar menos contrações e fornecer proteção contra o DM, quando comparadas às contrações EXC máximas, que fornecem um EP mais eficiente, mas induzem elevadas magnitudes de DM. Diferentemente do que se acredita com o DM induzido por contrações EXC, o EP resultante das contrações ISO máximas parece não se relacionar com o aumento do numero de sarcômeros em serie, ${ }^{12}$ mas sim por respostas relacionadas ao sistema inflamatório. Outro ponto levantado pelos mesmos autores é que o EP provocado por exercícios ISO é de curta duração e precisa de um intervalo de tempo ainda pouco Conexões: revista da Faculdade de Educação Física da UNICAMP, Campinas, v. 12, n. 3, p. 79-105, jul./set. 2014. 
investigado para se manifestar. Novos estudos precisam ser conduzidos para se entender melhor os mecanismos utilizados no EP induzido por contrações EXC e, principalmente, ISO.

Outra alternativa à realização de contrações EXC máximas como forma de proteção contra o DM que foi recentemente estudada é o aumento da temperatura muscular antes de exercícios que induzam DM. Nosaka et al. ${ }^{13}$ verificaram a atenuação dos sintomas de DM após exercícios EXC com aquecimento prévio da musculatura. Estratégias como esta podem ativar proteínas de choque térmico (HSP), que estão envolvidas na proteção das fibras musculares lesadas. No entanto, o EP induzido por exercícios EXC é mais forte que o induzido por esta estratégia.

Frente à grande diversidade de estratégias de proteção contra o DM causado por contrações EXC, o presente estudo teve como objetivo realizar uma revisão de literatura com a finalidade de apresentar o estado da arte no que diz respeito à proteção contra o DM a partir de atividades que não as contrações EXC máximas. Para tanto, foram realizadas buscas nas principais bases de dados (PubMed, Scielo, ISI-Web of Science) sobre o tema central, utilizando-se os termos "muscle damage", "protection", "isometric contractions", "flexibility", "stretching", "heating", entre outros. Os trabalhos encontrados com relevâncias ao assunto foram revisados e didaticamente expostos ao longo do texto em diferentes tópicos.

\section{DANO MUSCULAR E EFEITO PROTETOR}

O DM é um importante mediador de adaptações do músculo, pois, após as microlesões, o tecido muscular passa por um processo de recuperação e reestruturação, em que há um elevado aumento da síntese proteica. ${ }^{3}$ Vários autores têm conduzido estudos sobre as microlesões utilizando marcadores indiretos, que são úteis no diagnóstico e quantificação da magnitude do $\mathrm{DM}^{2-6}$ Os mais comuns são: perda da força muscular, manifestação da dor muscular de inicio tardio (DMIT), diminuição da amplitude de movimento articular (ADM), aumento da circunferência muscular (CIR) e atividade sérica ou plasmática da enzima creatina quinase $(\mathrm{CK}) .^{3-4,14-15}$ 
A diminuição da capacidade de produção de força muscular deve-se ao rompimento de ultraestruturas musculares que ocorre devido ao esforço excessivo dos sarcômeros que, por conta da sobreposição inadequada dos filamentos contrateis e das alterações no processo de excitação-contração., ${ }^{3,9,15}$ acabam por desorganizar ou romper-se, diminuindo ou anulando sua capacidade de produção de força. Imediatamente após a sessão, e nos 2 a 5 dias seguintes, os níveis de força permanecem abaixo dos valores antes do exercício., 15

A DMIT é a sensação de desconforto muscular, que apresenta um pico cerca de 24-48 horas após a indução de DM. Geralmente a dor se agrava quando a atividade realizada enfatiza mais contrações EXC do que CON. ${ }^{3,16,17}$ Toda vez que ocorre DM, o organismo desencadeia um processo inflamatório, com o qual objetiva-se recuperar o tecido lesionado liberando substâncias fundamentais para sinalização, limpeza e reconstrução da área afetada. Quatro fatores indicam a inflamação: calor, rubor, tumor e dor. Por conta da vasodilatação, tem-se um inchaço da região e maior concentração de sangue e, portanto, uma pequena elevação da temperatura. Acredita-se que a principal causa da DMIT é em decorrência do edema, que comprime as terminações nervosas levando à liberação de sinalizadores de dor, que indicam que aquela área não deve ser utilizada. ${ }^{18}$ Outros marcadores indiretos afetados pela inflamação, por conta do inchaço e da rigidez muscular, são a ADM e a CIR. ${ }^{2-6}$

A CK é considerada como o melhor indicador biomolecular da quebra da estrutura de uma célula muscular. O DM provoca desarranjos nas estruturas das células musculares, liberando enzimas próprias do meio intracelular para o sangue e aumentando sua atividade sérica ou plasmática nos dias após os exercícios EXC, apresentando seu pico de atividade geralmente entre 48-72 horas após a sessão e podendo permanecer elevada durante sete dias. $^{3}$

Já está bem estabelecido na literatura que, após sessões repetidas de exercícios EXC, de mesma intensidade e volume, há diminuição da magnitude do DM, bem como dos seus marcadores indiretos. Quando a musculatura é reparada e reestruturada após as lesões causadas, é gerada uma proteção parcial no músculo, protegendo-o de possíveis estresses que levem à ocorrência de novos danos. ${ }^{2,3,7,9,15,19,20}$ 
Efeito da carga repetida ou efeito protetor (EP) é um fenômeno muito estudado na literatura, e que ocorre logo após a recuperação do DM induzido por uma primeira sessão de contrações EXC, ${ }^{3,15}$ podendo durar entre 6 e 9 meses. ${ }^{5,21}$ Ainda que pouco se conheça a respeito dos mecanismos que causam este efeito, algumas teorias tentam o explicar. Em uma revisão, $\mathrm{McHugh}^{6}$ propôs três teorias de adaptações: a neural, a mecânica e a celular.

As teorias de adaptações neurais sugerem que após a ocorrência do DM, passa-se a recrutar mais unidades motoras lentas, bem como há melhora da sincronização de recrutamento das unidades motoras. A teoria das adaptações mecânicas aponta para o aumento da rigidez muscular dinâmica e passiva, e para o aumento do tecido conjuntivo como proteções musculares. Alguns estudos também apontam para uma concentração maior da proteína desmina nas miofibrilas, sendo essa uma das responsáveis pela orientação longitudinal e horizontal dos sarcômeros. ${ }^{7}$ A teoria celular sugere que ocorre aumento no número de sarcômeros em série, dividindo a tensão por unidade de sarcômero. As adaptações à resposta inflamatória e as adaptações para manter o acoplamento de excitação-contração também justificam esse EP. ${ }^{22}$

Embora já seja bem consolidado que contrações excêntricas máximas induzem o dano muscular e, por consequência, um EP, alguns estudos recentes vêm investigando estratégias alternativas de proteção contra o DM. Esses estudos se fundamentam no princípio de que talvez não seja necessário submeter um atleta ou praticante de atividade física a elevados níveis de DM para que esse apresente uma proteção contra o mesmo. Dessa forma, os tópicos a seguir apresentam 3 diferentes estratégias de proteção contra o DM que vêm sendo estudadas e parecem apresentar um EP considerável, mesmo na ausência de DM.

\section{TREINAMENTO DE FLEXIBILIDADE E ALONGAMENTO}

O alongamento estático vem sido estudado como um meio de se prevenir lesões musculares. Entretanto, não se encontra um consenso de que tal proteção realmente ocorra. ${ }^{23}$ Este conflito de informações pode ser explicado pelas limitações nas medidas de flexibilidade e do controle inadequado de certos fatores, como: tempo de estímulo, lesão anterior e tipo de lesão. ${ }^{24}$ É bem consistente na literatura que contrações excêntricas máximas (CEM) induzem DM mas, de acordo com MgHugh et al., ${ }^{25}$ ainda não está claro o 
porque de alguns indivíduos serem mais suscetíveis ao DM em relação a outros, quando submetidas a uma mesma sessão de exercícios EXC. Os autores colocam a possibilidade da flexibilidade, bem como a rigidez muscular, ser um fator que pode estar envolvido nesta diferença.

Armstrong et al. ${ }^{26}$ explanam que o DM ocorre quando a força muscular ativa está diminuindo enquanto a passiva está aumentando, o que sobrecarrega ainda mais as fibras musculares, pois as contrações EXC geram tensão por meio de elementos não contrateis, além dos contráteis, o que gera maior tensão por fibra muscular ativa devido ao fato desse tipo de contração recrutar menor número de unidades motoras. Estes pesquisadores afirmam que pessoas com músculos menos complacentes produzem uma força muscular passiva maior, o que pode gerar um maior DM.

Considerando esse conceito, McHugh et al. ${ }^{25}$ publicaram um artigo em que se teve como objetivo examinar o efeito da rigidez muscular passiva nos sintomas induzidos pelos exercícios EXC. Vinte sujeitos, homens e mulheres, participaram do estudo e, após terem a rigidez passiva do músculo medida, realizaram seis series de 10 contrações EXC a $60 \%$ da força máxima isométrica dos músculos isquiotibiais. Os indivíduos foram divididos em 3 grupos: complacente, normal e rígido. Os marcadores indiretos apresentam valores diferentes conforme a rigidez passiva apresentada. Verificou-se que a maior perda de força, dor, e CK foram do grupo rígido comparado com o grupo complacente. Frente a essas evidências, é possível considerar que músculos com maior rigidez passiva tendem a ser mais suscetíveis ao DM. Os autores propõem a explicação de que a tensão imposta pelo alongamento ativo da rigidez muscular seja transferida a partir do rígido complexo de tendão-aponeurose às fibras musculares, resultando em tensão miofribrilar. Em músculos complacentes o complexo tendão-aponeurose é capaz de absorver o alongamento, limitando, assim, a tensão miofibrilar. Dessa forma, a rigidez passiva parece refletir a extensibilidade do tecido conjuntivo e das fibras musculares.

Alguns estudos comparam a magnitude do DM entre crianças e adultos, pois estas, segundo Soares et al., ${ }^{27}$ parecem sofrer menos DM comparadas com homens jovens, após uma sessão de treinamento de força. Em seu estudo, em que dez meninos e 10 homens foram submetidos a duas sessões de oito series de 10 saltos pliométricos, observou-se que, 
nos meninos, os sintomas eram menos intensos que nos adultos, e os resultados indicaram, também, que as crianças têm maior flexibilidade, o que levaria a uma menor tensão dos sarcômeros durante o exercício EXC, além de possuírem menor número de fibras de contração rápida. Marginson e Eston ${ }^{28}$ demonstram que a extensibilidade (flexibilidade passiva) do quadríceps é significantemente maior em meninos quando comparados com homens.

Marginson et al. ${ }^{9}$ também investigaram a diferença da magnitude do DM entre dez meninos e dez homens, sendo que todos eles executaram duas sessões de oito series de dez exercícios pliométricos (saltos). Os resultados mostraram que o DM foi evidente em todos os sintomas examinados em homens, e somente na dor para os meninos, levando os autores a crer que os sintomas mais intensos de DM foram diagnosticados nos homens. Como esperado, a extensão do quadril foi maior nos meninos em comparação com os homens. Isso pode ter contribuído para a diferença nas curvas de comprimento-tensão. $\mathrm{O}$ comprimento muscular é um importante fator de moderação na magnitude do DM, pois exercícios realizados em comprimentos musculares maiores levam a maiores níveis de DM.

LaRoche e Connoly ${ }^{23}$ realizaram um estudo em que o alongamento foi usado numa tentativa de melhorar o desempenho e reduzir o risco de lesão muscular. Participaram do estudo vinte e nove homens, sendo estes designados aleatoriamente para os grupos: alongamento estático, alongamento dinâmico ou controle. Todos os grupos realizaram testes de avaliação durante quatro dias, em que quatro extensões da amplitude máxima de movimento (de forma passiva) foram realizadas, bem como a mensuração de rigidez, ADM, absorção de trabalho, pico de torque e dor. Os grupos experimentais realizaram os treinos de seus respectivos alongamentos três vezes na semana durante quatro semanas, após este treino realizou-se novamente os quatro dias de teste. Após o primeiro dia de avaliação, todos os grupos realizaram CE e nos três dias seguintes mediram-se marcadores de DM.

Após as quatro semanas de alongamento moderado, o grupo experimental não apresentou mudanças significantes na rigidez muscular, na absorção do trabalho e na DMIT. Entretanto, verificaram-se alterações significantes na ADM, podendo assim explicar o 
aumento no pico de torque, o que indicaria que os participantes foram capazes de aguentar maiores tensões musculares. Entretanto as mudanças nas propriedades elásticas não foram duradouras, o que se esperava para explicar o aumento da ADM. Ainda que um programa de treino de resistência possa proporcionar um EP maior que o observado por um treino de flexibilidade moderado, tanto o alongamento estático quanto o dinâmico aumentam a $\mathrm{ADM}$ e a tolerância ao alongamento após exercícios EXC. Os autores propuseram que novas pesquisas sejam feitas, com duração do treinamento maior do que 8 semanas, pois a intensidade e o volume podem produzir alterações na rigidez e na capacidade de absorção de trabalho do músculo esquelético.

Eston et al. ${ }^{29}$ avaliaram o efeito do treinamento da flexibilidade, por meio da facilitação neuromuscular proprioceptiva (FNP), nos marcadores indiretos do DM. Os autores tinham como hipótese que os participantes que realizaram o treinamento de flexibilidade teriam seus sintomas de DM atenuados. Quarenta homens participaram do estudo, o grupo experimental realizou 2 sessões de treino de flexibilidade por semana, por meio da FNP, durante 5 semanas. Em seguida, os grupos controle e experimental realizaram 6 sessões de 10 contrações EXC isocinéticas dos flexores do joelho do membro dominante. O treinamento de FNP provocou um aumento na flexibilidade e levou a uma pequena proteção contra a queda de força num comprimento longo do músculo. Entretanto, nenhuma proteção foi observada contra a dor, rigidez ou perda de força muscular num comprimento curto do músculo. O treinamento de flexibilidade não parece ter induzido um EP neste estudo, o que contraria os achados de McHugh et al. ${ }^{25}$. Entretanto, existem varias explicações possíveis, como: a intensidade das contrações (submáximas e máximas), gênero dos sujeitos e o desenho experimental (distribuição aleatória e desenho transversal). Mais pesquisas devem ser conduzidas para analisar os potenciais do EP conferido por treinamento de flexibilidade sobre os sintomas de DM.

Embora os estudos de LaRoche e Connoly ${ }^{23}$ e Eston et al. ${ }^{29}$ tenham sido os primeiros a investigar os efeitos do treinamento de flexibilidade com o intuito de conferir um EP, estes não sugerem com muita propriedade que este tipo de treinamento reduz os sintomas do DM induzido por contrações EXC. Chen et al., ${ }^{8}$ baseados em estudos de modelo animal, hipotetizaram que o treinamento de flexibilidade geraria mudanças no ângulo ótimo de produção de força do músculo (aumentando a angulação na qual ele ocorre), o que poderia 
atenuar a magnitude do DM induzido pelo exercício EXC. Sendo assim, o objetivo apresentado pelos autores foi verificar se 8 semanas de alongamento estático e FNP dos flexores do joelho tornariam os músculos menos suscetíveis ao DM e também conferir se o treinamento de FNP seria mais eficaz do que o alongamento estático na atenuação do DM.

Trinta homens participaram do estudo e foram divididos em três grupos: controle, alongamento estático e FNP. Os grupos experimentais realizaram programas de treinamento de três sessões semanais de suas respectivas modalidades ao longo de 8 semanas seguidas. Quatro dias após o final dos treinamentos, foram realizadas contrações EXC. O grupo controle se manteve inativo nesse período, apenas realizando as contrações EXC. Marcadores indiretos de DM foram coletados um dia antes, imediatamente antes das contrações EXC, e imediatamente, e de 1 a 5 dias após as mesmas. Este estudo foi o primeiro a mostrar que as atividades de pré-condicionamento (alongamento passivo e FNP) foram capazes de atenuar o DM induzido por CE. Ambos os grupos experimentais apresentaram mudanças atenuadas nas variáveis $\mathrm{ADM}$, força $\mathrm{CON}$, ângulo ótimo de produção de força, DMIT, e CK após os exercícios EXC, quando comparados com o grupo controle. Não foram identificadas diferenças no EP conferido pelas duas modalidades de treino de flexibilidade.

Os resultados encontrados vão de acordo com o estudo de McHugh et al. ${ }^{25}$, pois mostram que músculos flexíveis são menos suscetíveis a DM induzido por CE. Entretanto não vão de acordo com os achados de LaRoche e Connoly ${ }^{23}$ e Eston et al. ${ }^{29}$, pois o EP encontrado por estes autores foi mais fraco em relação ao encontrado pelos demais. Uma explicação plausível para essa diferença de resultados é que talvez a duração do treino de flexibilidade e o tempo total exposto aos alongamentos não foram suficientes para produzir um EP potente.

Baseado nos dados apresentados seria razoável assumir que a flexibilidade do músculo seja um fator que determina a intensidade dos DM induzido por contrações EXC. Entretanto, estudos devem ser realizados para melhor se entender o mecanismo pelo qual a flexibilidade confere o EP. Faz-se necessário investigar: a) as mudanças no comportamento do músculo-tendão durante as $\mathrm{CE}$ depois de treinamento de flexibilidade; b) as mudanças no numero de sarcômeros em série após o treinamento de flexibilidade e como isso afeta o 
comportamento do sarcômero durante as contrações EXC; e c) a diferença na flexibilidade entre os sexos e idades.

\section{CONTRAÇÕES ISOMÉTRICAS}

Outra estratégia que vem sendo investigada para conferir um EP contra o DM é a realização prévia de contrações isométricas máximas (CIM). Essa estratégia foi investigada pela primeira vez em um estudo de Clarkson et al., ${ }^{30}$ em que 11 homens realizaram duas sessões, espaçadas por 1 semana, de exercícios ISO. O grupo A realizou 40 CIM com duração de 10 segundos e com descanso de 20 segundos entre cada contração. O grupo B realizou o mesmo numero de contrações com a mesma duração que o grupo A, entretanto com um intervalo de 5 segundos. Uma semana mais tarde, ambos os grupos realizaram outra sessão de exercícios igual à do grupo A.O aumento da atividade da CK após a primeira sessão foi significantemente maior para o grupo A (143\%) comparado com o grupo B (52\%). O que inicialmente levou os autores a concluírem que este aumento deu-se pelo alto nível de tensão, mas, após a segunda sessão verificou-se uma redução da CK, levando-se a uma conclusão de que a resposta da CK não estava relacionada com a tensão gerada.

Koh e Brooks $^{31}$ conduziram um estudo em que tinham como hipótese que CIM ou alongamentos passivos não induziriam dano nem regeneração muscular, portanto não produziriam EP. Os sujeitos da pesquisam foram ratos machos, que, por estimulação elétrica, realizaram 75 contrações máximas dos extensores longos dos dedos. Dividiu-se os ratos em três grupos: contração alongada, músculo relaxado (alongamento passivo) e CIM (no comprimento ótimo do músculo). Duas semanas após as atividades de précondicionamento todos os grupos realizariam 75 CEM. Após 10 minutos e três dias, o grupo que realizou as CIM não apresentou quedas significantes de força. Três dias após a realização de CEM, não se encontrou uma porcentagem significativa de alteração em marcadores de DM do grupo que realizou as CIM quando comparados com os músculos destreinados. Ao contrário do que os autores acreditavam, os resultados demonstraram que nem contrações alongadas, nem a degeneração e/ou regeneração de fibras musculares são necessárias para induzir um EP contra ações EXC. E que mesmo treinamentos com ações ISO, bem como com o músculo relaxado, podem provocar uma proteção muscular. Os autores relatam que os músculos submetidos a alongamentos passivos mostraram Conexões: revista da Faculdade de Educação Física da UNICAMP, Campinas, v. 12, n. 3, p. 79-105, jul./set. 2014. 
evidencias de dilatação dos vasos sanguíneos e edema após 3 dias, o que sugere um indicio de dano nos vasos ou no tecido conjuntivo. Entretanto, consideraram que os dados precisavam ser reproduzidos e mais estudos deveriam ser realizados para investigar se estes fatores desempenhariam um papel de indução de EP.

A proteção provocada por CI é menore do que as provocadas por CE, o que deixa claro que para se conseguir maiores resultados de proteção ou magnitude do mesmo precise-se lesionar o músculo, ou seja, os estímulos precisam ser mais fortes. Koh \& Brooks ${ }^{31}$ também sugerem que outros estudos sejam feitos em que haja estímulos ISO maiores para que se observe se um maior EP será produzido.

Seguindo o mesmo protocolo de Koh e Brooks, ${ }^{31}$ Pizza et al. ${ }^{18}$ testaram a hipótese de que as CIM e alongamentos passivos, mesmo não causando dano, podem aumentar a concentração de células inflamatórias musculares que, com a realização subsequente de CEM, pode ser atenuada. Para justificar esse raciocínio, os autores justificaram que os neutrófilos são as primeiras células a aparecer no músculo e acredita-se que estes retardem a regeneração, agravando a lesão inicial, pois liberam radicais livres, que provocam uma alteração nas membranas celulares, causando uma nova lesão como consequência de um processo inflamatório nas fibras musculares, ${ }^{32}$ bem como proteases, que são enzimas que quebram ligações peptídicas entre os aminoácidos das proteínas. Entretanto, os neutrófilos também podem facilitar a regeneração pela remoção de detritos na área lesionada por meio da fagocitose e da ativação de células satélites. A contribuição de neutrófilos para esses acontecimentos ainda não é bem compreendida. Os macrófagos também são células que ajudam na restauração das áreas lesionadas por meio da fagocitose de restos de tecido e por causar proliferação dos mioblastos. ${ }^{33}$ As CIM e os alongamentos passivos geraram, agudamente, um aumento significativo de neutrófilos, mas não de macrófagos.

No estudo de Pizza et al., ${ }^{18}$ ratos foram separados em três grupos, sendo que todos realizaram contrações EXC máximas, precedidas por alongamento passivo, CIM e contrações EXC, de acordo com cada um dos grupos. O grupo que realização contrações EXC teve um aumento de neutrófilos maior que os outros grupos, e um aumento significativo na concentração de macrófagos. Após a sessão de indução de DM por meio de contrações EXC, todos os grupos obtiveram uma redução significativa das células Conexões: revista da Faculdade de Educação Física da UNICAMP, Campinas, v. 12, n. 3, p. 79-105, jul./set. 2014. 
inflamatórias. Os autores afirmam que os neutrófilos aumentam em concentração mesmo com a musculatura não apresentando danos histológicos e funcionais, ao contrario do que os macrófagos apresentam, pois estes apenas se elevaram quando os sinais de danos foram evidentes. Ao analisar que após a sessão de contrações EXC, os grupos de CIM e o de alongamento passivo apresentaram um EP, pode-se dizer que as células inflamatórias poderiam contribuir para uma adaptação ao DM, conferindo um FP. Ainda assim, uma investigação mais aprofundada precisa ser realizada para entender os fatores que atraem os neutrófilos para os músculos esqueléticos lesionados e não lesionados.

Os primeiros artigos publicados com o intuito de investigar sistematicamente as magnitudes do EP induzido por CI antes de CE são recentes. ${ }^{10-12}$ Esses estudos foram publicados pelo mesmo grupo de pesquisa que identificou, em estudos feitos anteriormente, que a recuperação das funções musculares era mais rápida quando os sujeitos realizavam contrações isométricas em sessões de familiarização ${ }^{34-35}$ do que aqueles estudos em que não haviam familiarizações prévias. ${ }^{36-37}$

Sendo assim, o primeiro artigo ${ }^{10}$ teve como objetivo investigar qual seria a magnitude do FP conferido pela realização de CIM antes de uma sessão de CEM nos flexores do cotovelo. Trinta e nove sujeitos homens jovens foram divididos em três grupos: controle e experimentais (2 e $10 \mathrm{CIM}$ ), realizando os respectivos protocolos de CIM dois dias antes das 30 CEM, adotando-se um comprimento longo do músculo $\left(20^{\circ}\right)$ durante as contrações. Os resultados mostraram que os números de CIM usadas pelo estudo não levaram a alterações nos marcadores de DM, o que difere de um estudo apresentado por Phillippou et al. ${ }^{38}$, que mostrou que duas sessões de 25 CIM causaram mudanças significantes em marcadores de DM. Ainda assim percebeu-se, após as CEM, que os grupos experimentais tiveram uma mudança significativa na velocidade de recuperação da força muscular concêntrica máxima (MVC-CON), sendo esta mais acelerada para o grupo que realizou 10 CIM quando comparados com os dois outros grupos e mais acelerada para o grupo que realizou 2 CIM em relação ao grupo controle. A magnitude das alterações dos marcadores de DM também foi significantemente menor para os grupos experimentais em relação ao grupo controle. Esses dados levaram os autores a concluir que a magnitude do EP não é necessariamente proporcional ao numero de CIM. Este foi o primeiro artigo a demonstrar 
categoricamente que a realização de CIM dois dias antes de CEM pode atenuar os sintomas de DM.

O segundo artigo publicado por Chen et al. ${ }^{12}$ teve como intuito identificar a duração do EP conferido por CIM realizadas em duas amplitudes diferentes $\left(20^{\circ}\right.$ e $\left.90^{\circ}\right)$, e comparar a magnitude deste com o EP conferido por CEM ou contrações EXC submáximas (CES). Os autores alegaram que não haveriam estudos investigando as diferenças de magnitudes do EP conferido por CIM com diferentes angulações. Sendo assim, os autores compararam a eficiência de cinco protocolos de pré-condicionamento na manifestação do EP contra o DM induzido por CEM. Os protocolos adotados foram: a realização de CEM, realização de CEM submáximas em duas diferentes intensidades (10\% e $20 \%$ da força excêntrica máxima) e CIM em duas diferentes amplitudes $\left(20^{\circ}\right.$ e $\left.90^{\circ}\right)$. Para tanto, sessenta e cinco sujeitos do sexo masculino foram divididos em cinco grupos (todos compostos por 13 indivíduos): $\mathrm{CEM}, 10 \% \mathrm{EXC}, 20 \% \mathrm{EXC}, 90^{\circ} \mathrm{CIM}$ e $20^{\circ} \mathrm{CIM}$. Os três primeiros grupos realizaram $30 \mathrm{CE}$ dos flexores do cotovelo, os outros dois grupos realizaram $30 \mathrm{CIM}$, também dos flexores do cotovelo. Três semanas após a realização desses protocolos, todos os grupos realizaram 30 CEM.

Após as atividades de pré-condicionamento, todos os grupos obtiveram mudanças significantes marcadores de força e dor muscular. Após a realização das CEM, três semanas após as atividades de pré-condicionamento, todos os grupos, exceto o grupo CEM que não obteve diferenças significantes na $\mathrm{CK}$ e $\mathrm{Mb}$, tiveram mudanças significantes em todos os marcadores de DM. As maiores alterações dos marcadores de DM nessa condição, quando comparados os grupos, foram apresentadas pelos grupos $90^{\circ} \mathrm{CIM}$ e $10 \%$ EXC. Os grupos CEM, 20\% EXC e $20^{\circ} \mathrm{CIM}$, por sua vez, apresentaram menores alterações nos marcadores de DM e, portanto, maiores FP. Dessa forma, os autores conseguiram demonstrar que apenas os grupos $\mathrm{CEM}, 20^{\circ} \mathrm{CIM}$ e $20 \%$ EXC apresentaram atenuação dos marcadores indiretos de DM após a realização de CEM. Os resultados apresentados pelos outros dois grupos eram similares aos encontrados para o grupo controle.

Entretanto, uma das hipóteses dos autores não se confirmou no estudo, pois os resultados demonstraram que o grupo $20^{\circ} \mathrm{CIM}$ (27\%-63\% de proteção conferida) apresentou um EP de maior magnitude do que o grupo 20\% EXC (17\%-55\%). Os autores concluíram, então, 
que CIM realizadas num comprimento longo do músculo induz melhor EP que contrações EXC de baixa intensidade. Fica claro, então, que CIM realizadas com um comprimento de músculos maior podem levar à manifestação de um EP que não é conferido quando essas são realizadas em uma angulação encurtada. Ademais, os autores destacam que o grupo $20^{\circ}$ CIM foi o único a manifestar mudanças no ângulo ótimo de produção de força (AO) antes da realização das CEM e foi o único, após as CEM, a apresentar atenuação de todos os marcadores indiretos de DM exceto o AO. Esses dados levam a crer que houve um aumento do número sarcômeros em serie, o que pode ter contribuído para a manifestação do EP.

O terceiro e mais recente artigo a investigar o uso de CIM como estratégia de proteção contra o $\mathrm{DM}^{11}$ teve como objetivo verificar se os marcadores de DM são atenuados quando os exercícios EXC máximos são realizados imediatamente, 2, 4 ou 7 dias após a realização de 2 CIM $\left(20^{\circ}\right)$. Sendo assim, 65 sujeitos homens foram divididos em 5 grupos de acordo com o momento em que realizaram as CIM: a) imediatamente antes das CEM (Od); b) 2 dias antes das CEM (2d); c) 4 dias antes das CEM (4d); d) 7 dias antes das CEM (7d) e e) controle. Nenhum dos grupos experimentais apresentou mudanças significantes em marcadores de DM após as CIM. Os grupos 2d e 4d obtiveram os melhores resultados em todas as variáveis, apresentando menores mudanças para o $\mathrm{AO}$, melhor recuperação da força $\mathrm{CON}$ voluntaria máxima, menores concentrações de $\mathrm{CK}$ e $\mathrm{Mb}$, menores aumentos da CIR, menor grau de desenvolvimento de dor quando comparados com os outros grupos. Além disso, quando comparados entre si, o grupo $2 \mathrm{~d}$ apresentou resultados melhores do que o grupo $4 \mathrm{~d}$ com a exceção do marcador $\mathrm{AO}$. Os grupos controle, $0 \mathrm{~d}$ e $7 \mathrm{~d}$ não apresentaram resultados diferentes entre si.

Os resultados obtidos demonstraram que o EP se manifestou até o quarto dia após as CIM, mas não se manteve até o sétimo dia. Imediatamente após também não se verificou um EP, o que pode indicar que o EP produzido por CIM necessita de um período de tempo para se manifestar. Os autores ressaltam que não houve um grupo que realizasse os exercícios EXC após 1 dia das CIM, o que não permite afirmar se o EP se manifestaria 24 horas após as CIM nem fazer comparações de magnitudes entre 1 e 2 dias. 
Baseado nos estudos que investigaram a influência de CIM na proteção contra o DM, alguns considerações podem ser feitas: 1) Estudos que investigam o DM em que seja necessária a familiarização devem se atentar para que esta ocorra 1 semana antes das CEM, pois podem afetar a magnitude do DM; 2) Se as CIM forem realizadas em um comprimento curto do músculo, os efeitos serão mínimos; 3) CIM podem ser utilizadas como exercícios de pré-condicionamento quando se deseja atenuar o DM pelo exercício.

\section{AQUECIMENTO ATIVO E PASSIVO}

A rigidez passiva tem sido apontada como um dos mecanismos responsáveis pela magnitude do DM. Acredita-se que, aumentando a temperatura muscular induzida por exercícios de aquecimento, seria possível, através da extensibilidade do músculo (capacidade do músculo de alongar ou esticar além do comprimento em repouso), tecido conjuntivo e aumento da viscosidade muscular, tornar o músculo menos suscetível a DM. ${ }^{39-40}$ Além do aumento da temperatura influenciar na complacência da musculatura, outros fatores também são afetados, como as propriedades viscoesqueléticas da unidade musculotendínea, que incluem modificações nos reflexos de estiramento durante o aquecimento, podendo proteger o músculo por conta da redução da rigidez. ${ }^{13}$ McNair e Stanley $^{41}$ alegam que a redução da rigidez ativa pode ser alcançada por meio de aquecimentos específicos à atividade a ser realizada. Frente a isso, uma estratégia de prevenção contra o DM que vem sendo bastante estudada é o aquecimento prévio. É comum que séries de aquecimento sejam implementadas, não só em treinamento de atletas, mas também com o intuito de evitar lesões. ${ }^{13,42}$

Rodenburg et al. ${ }^{39}$ estudaram a combinação de exercícios de aquecimento, alongamento e massagem com a intenção de diminuir a DMIT. Cinquenta pessoas participaram do estudo, e foram divididas em dois grupos: controle e experimental. Todos os indivíduos realizaram os exercícios com os flexores do antebraço por 30 minutos. O grupo experimental realizou um aquecimento e alguns exercícios de alongamento, seguidos por uma sessão de exercícios EXC e, subsequentemente, o grupo muscular utilizado foi massageado. O grupo controle realizou apenas os exercícios EXC. Foram identificadas alterações significantes nos marcadores força máxima, ângulo de flexão do cotovelo e CK após os exercícios EXC para ambos os grupos. Entretanto, esses resultados não foram significantemente diferentes 
entre os grupos. Portanto, a combinação de aquecimento, alongamentos e massagem se mostrou ineficaz na prevenção contra o DM neste estudo.

Um estudo conduzido por Nosaka e Clarkson ${ }^{43}$ investigou se um exercício CON fatigante realizado imediatamente antes do exercício EXC aumentaria a magnitude do DM quando comparado com o DM sofrido após uma sessão de exercício excêntrico sem a realização de atividade prévia. Nove mulheres participaram do estudo e executaram 12 CEM nos flexores do cotovelo com ambos os braços. Entretanto, um dos braços passou por um protocolo de 100 contrações isocinéticas CON antes da realização do das CEM. Todos os marcadores de DM mudaram significativamente em ambas as condições, e houve diferença significativa entre elas. Após as CEM verificou-se que o braço que fez as contrações CON apresentou um FP, apresentando um menor nível de dor, recuperação mais rápida da força ISO máxima, menor redução do ângulo da articulação do cotovelo relaxado e menor aumento da CIR do braço e da CK, indicando que o DM foi atenuado pela realização prévia de exercício CON. Esses dados indicam que a realização de exercícios CON antes dos exercícios EXC pode ter elevado a temperatura muscular, diminuindo o DM. O resultado desse estudo indica que, durante o aquecimento, talvez seja necessário um enfoque em músculos específicos, uma vez que aquecimentos generalizados (corrida) não parecem afetar a rigidez passiva do músculo. ${ }^{44}$ Se a rigidez ativa for, de fato, um mecanismo importante por trás da magnitude do DM, um aquecimento específico poderia ajudar a reduzir a severidade do DM.

Com o intuito de melhor entender qual a temperatura ideal para prevenir lesões, Evans et al. ${ }^{45}$ se propuseram a testar os efeitos do aquecimento sobre o DM resultante de exercícios EXC comparando os efeitos de aquecimentos passivos e ativos antes da realização de CEM. Quarenta e três sujeitos participaram da pesquisa (16 homens e 27 mulheres), e foram divididos em 5 grupos: Aquecimento passivo com baixa temperatura $(n=10)$; Aquecimento passivo com alta temperatura $(n=4)$; Aquecimento ativo por meio de contrações CON (n=9); Controle (i.e., realização das CEM sem aquecimento prévio) (n= 10); Apenas aquecimento passivo de alta temperatura, sem a realização das CEM ( $n=10)$. $\mathrm{O}$ aquecimento passivo foi realizado por meio de diatermia de ondas curtas pulsantes. Marcadores de DM foram coletados 24, 48, 72 e 168 horas após a realização das CEM. Os resultados demonstraram que o aquecimento passivo, realizado antes de CEM, pode ser 
mais benéfico que o aquecimento ativo ou a não realização de aquecimentos em atenuar o inchaço muscular. Entretanto, não foram identificadas evidências de que o aquecimento previne ou atenua a perda de forca, amplitude de movimento e dor. Os autores sugerem que mais estudos sejam feitos na área, pois houve grande variabilidade inter-individual nas respostas ao exercício EXC.

Um estudo conduzido por Nosaka et al. ${ }^{13}$ buscou investigar se o contrario era verdadeiro: o resfriamento do músculo acentuaria o DM. Para tanto, 20 voluntárias foram divididas em dois grupos de acordo com a estratégia adotada antes da indução de DM: aquecimento e resfriamento. Após as intervenções de aquecimento/resfriamento, ambos os grupos realizaram 12 CEM, como forma de indução de DM. Os achados desse estudo foram que o resfriamento não aumenta a magnitude do DM, tampouco o aquecimento muscular.

Ainda que os mecanismos do EP não estejam bem esclarecidos, uma das teorias que tentam explicar este fenômeno é a das proteínas de choque térmico (HSPs), que desempenham um papel importante em situações que envolvam estresse. Essas proteínas podem estar envolvidas na proteção das fibras musculares esqueléticas contra estímulos que induzem DM. ${ }^{46}$ Sabe-se que a concentração delas aumenta quando a temperatura do músculo é elevada acima de $40^{\circ} \mathrm{C} .{ }^{47-48}$ Diante desse quadro, Nosaka et al. ${ }^{14}$ realizaram o primeiro estudo em humanos em que a temperatura muscular foi elevada até $40^{\circ} \mathrm{C} 16-20 \mathrm{~h}$ antes da realização de CEM. Quinze sujeitos homens participaram do estudo e um de seus braços foi submetido a hipertermia passiva por 20 minutos e, após um dia, à um sessão de 24 CEM. O braço contralateral serviu como controle, não passando por hipertermia e realizando 24 CEM. Os resultados apontaram para alterações em todos os marcadores de DM coletados em ambos os braços. Entretanto, o grupo experimental apresentou um FP evidenciado pela recuperação mais rápida da força de contração ISO voluntária máxima, menores mudanças na ADM e menor desenvolvimento de dor. Frente a esses dados, os autores levantaram a hipótese de que as HSPs possam ter tido influencia nesse EP, entretanto, este estudo não mensurou a quantidade de HSPs para poder afirmar se elas realmente tiveram relação com os resultados encontrados ou se o aquecimento causado influenciou outros sistemas do corpo como os sistemas central e imunológico. Estudos anteriores, como os de McArdle et al. ${ }^{49}$ e Miyabara et al., ${ }^{50}$ mostram que a concentração elevada de HSP70 em ratos diminuiu a queda de força e aumentou sua velocidade de Conexões: revista da Faculdade de Educação Física da UNICAMP, Campinas, v. 12, n. 3, p. 79-105, jul./set. 2014. 
recuperação, havendo um maior numero de fibras musculares intactas após a realização de CEM, quando comparado a condições basais.

Considerando os estudos acima expostos, pode-se considerar que, embora o mecanismo fisiológico ainda não tenha sido precisamente identificado, o aquecimento prévio parece agir de forma a conferir um EP contra o DM induzido pelo exercício. Ainda se fazem necessários estudos que investiguem: 1) Diferentes tempos de exposição ao calor previamente ao estimula danificador; 2) A influência de diferentes intensidades de aquecimento passivo e ativo no EP conferido; 3) A relação das HSPs com a hipertermia na prevenção contra o DM induzido pelo exercício.

\section{CONCLUSÃO}

O EP conferido por atividades de pré-condicionamento como aquecimento, treinamento de flexibilidade e realização de CIM parece não ser tão intenso como aquele conferido por CEM. Entretanto, ao se pensar em estratégias práticas, essas atividades de précondicionamento podem ser consideradas como alternativa eficiente para se evitar o DM sem que o músculo tenha que passar por um estresse elevado. É evidente que o EP conferido por essas atividades não protege o músculo por um longo período de tempo, entretanto, essas estratégias podem ser empregadas de maneira simples e rápida, além de dispensar a necessidade de recursos caros e de complicada logística como dinamômetros isocinéticos.

O uso de treinamento de flexibilidade ou alongamento prévio como estratégia para conferir um EP ainda precisa ser investigado mais profundamente, principalmente no que concerne a implementação de treinamentos que durem mais que 8 semanas, pois a intensidade e o volume parecem ser importantes mediadores das alterações na rigidez e na capacidade de absorção de trabalho do músculo esquelético. Além disso, novos estudos se fazem necessários para identificar com maior propriedade os mecanismos fisiológicos por trás do EP induzido pela complacência muscular.

A realização de CIM em um período de até quatro dias antes da realização de CEM parece conferir um EP considerável mesmo quando poucas contrações são realizadas. Os estudos apresentados demonstram que a realização das CIM num comprimento longo do músculo 
induz melhor EP que contrações EXC de baixa intensidade. Seria interessante a realização de estudos em que houvesse a mistura de CIM e contrações EXC submáximas para verificar se há o surgimento de um EP mais efetivo. Um maior número de estudos também é necessário para fundamentar os mecanismos fisiológicos por trás do EP conferido por CIM, partindo da teoria de que essas levam a uma resposta imunológica atenuada a uma sessão danificadora.

$\mathrm{O}$ aquecimento muscular antes da realização de CEM ainda precisa ser mais profundamente estudado, mesmo considerando que alguns estudos tenham apontado para a manifestação de um EP após essa estratégia. É necessário verificar o tempo de exposição ao aquecimento e qual a melhor forma para se aquecer a musculatura. Novos estudos com enfoque nas HSPs são necessários para que se possa consolidar um corpo de conhecimento acerca da relação dessas com o EP induzido pela hipertermia muscular.

\section{REFERÊNCIAS}

${ }^{1}$ MORITANI, T.; DE VRIES, H. A. Neural factors versus hypertrophy in the time course of muscle strength gain. American Journal of Physical Medicine e Rehabilitation, Baltimore, v. 58, n. 3, p. 115-130, june 1979.

${ }^{2}$ CLARKSON, P. M.; HUBAL, M. J. Exercise-induced muscle damage in humans. American Journal of Physical Medicine e Rehabilitation, Baltimore, v. 81, n. 11, p. S53-S69, nov. 2002.

${ }^{3}$ BRENTANO, M. A.; KRUEL, L. F. M. A review on strength exercise-induced muscle damage: applications, adaptation mechanisms and limitations. Journal of Sport Medicine and Physical Fitness, Torino, v. 51, n. 1, p. 1-10, mar. 2011.

${ }^{4}$ LIMA, L. C. R.; DENADAI, B. S. Efeito protetor após sessões de exercício excêntrico: comparação entre membros superiores e inferiores. Motriz, Rio Claro, v. 17, n. 4, p.738747, out. 2011.

Conexões: revista da Faculdade de Educação Física da UNICAMP, Campinas, v. 12, n. 3, p. 79-105, jul./set. 2014. 
${ }^{5}$ BARROSO, R.; TRICOLI, V.; UGRINOWITSCH, C. Adaptações neurais e morfológicas ao treinamento de força com ações excêntricas. Revista Brasileira de Ciência e Movimento, Brasília, v. 13, n. 2, p.111-122, ago. 2005.

${ }^{6} \mathrm{McHUGH}$, M.P. Recent advances in the understanding of the repeated bout effect: the protective effect against muscle damage from a single bout of eccentric exercise. Scandinavia Journal of Medicine and Science in Sports, Oxford, v. 13, n. 2, p. 88-97, apr. 2003.

${ }^{7}$ NOSAKA, K. et al. Partial Protection against Muscle Damage by Eccentric Actions at Short Muscle Lengths. Medicine and Science in Sport and Exercise, Hagerstown, p. 746-753, may 2005.

${ }^{8} \mathrm{CHEN}, \mathrm{CH}$. et al. Effects of flexibility training on eccentric exercise-induced muscle damage. Medicine and Science in Sport and Exercise, Hagerstown, v. 43, n. 3, p. 491500, mar. 2011.

${ }^{9}$ MARGINSON, V.F. et al. Comparison of the symptoms of exercise-induced muscle damage following an initial and repeated bout of plyometric exercise in men and boys. Journal of Applied Physiology, Bethesda, v. 99, p. 1174-1181, apr. 2005.

${ }^{10} \mathrm{CHEN}, \mathrm{T}$. C. et al. Attenuation of eccentric exercise induced muscle damage by preconditioning exercises. Medicine and Science in Sports and Exercise, Hagerstown, v. 44, n. 11, p. 2090-2098, nov. 2012.

${ }^{11}$ CHEN, T.C. et al.. Effect of two maximal isometric contractions on eccentric exerciseinduced muscle damage of the elbow flexors. European Journal of Applied Physiology, Heidelberg, v. 113, p. 1545-1554, june 2013.

${ }^{12} \mathrm{CHEN}$, H.L. et al. Two maximal isometric contractions attenuate the magnitude of eccentric exercise-induced muscle damage. Applied Physiology Nutrition Metabolism, Toronto, v. 37, p. 680-689, ago. 2012. 
${ }^{13}$ NOSAKA, K. et al. Influence of pre-exercise muscle temperature on responses to eccentric exercise. Journal of Atthletic Training, Dallas, v. 39, p.132-137, june 2004.

${ }^{14}$ NOSAKA, K. et al. Attenuation of muscle damage by preconditioning with muscle hyperthermia 1-day prior to eccentric exercise. European Journal of Physiology, Heidelberg, p. 183-192, nov. 2007.

${ }^{15}$ GLEESON, N. et al. Effects of prior concentric training on eccentric exercise induced muscle damage. British Journal of Sports Medicine, Loughborough, p. 119-125, apr. 2003.

${ }^{16}$ FOSCHINI, D.; PRESTES, J.; CHARRO, M. A. Relação entre exercício físico, dano muscular e dor muscular de início tardio. Revista Brasileira de Cineantropometria e Desenvolvimento Humano, Florianópolis, v. 9, n. 1, p.101-106, mar. 2007.

${ }^{17}$ TRICOLI, V. Mecanismos envolvidos na etiologia da dor muscular tardia. Revista Brasileira de Ciência e Movimento, Brasília, v. 9, n. 2, p. 39-44, apr. 2001.

${ }^{18}$ PIZZA, F.X. et al. Muscle inflammatory cells after passive stretches, isometric contractions, and lengthening contractions. Journal of Applied Physiology, Bethesda, v. 92, n. 5, p. 1873-1878, may 2002.

${ }^{19}$ NOSAKA, K.; NEWTON, M.; SACCO, P. Responses of human elbow flexor muscles to electrically stimulated forced lengthening exercise. Acta Physiologica Scandinavica, Copenhagen, v.174, p. 137-145, feb. 2002.

${ }^{20}$ HOWATSON, G.; VAN SOMEREN, K.; HORTOBÁGYI, T. Repeated bout effect after maximal eccentric exercise. International Journal of Sports Medicine, Stuttgart, v. 28, n. 7, p. 557-563, july 2007 .

${ }^{21}$ NOSAKA, K.; SAKAMOTO, K.; NEWTON, M.; SACCO, P. How long does the protective effect on eccentric exercise-induced muscle damage last? Medicine and Science in Sports and Exercise, Hagerstown, v. 33, n. 9, p. 1490-1495, sept. 2001. 
${ }^{22}$ MORGAN, D.L. New insight into the behaviour of muscle during active lengthening. Biophysical Journal, Nova York, v. 57, p. 209-221, feb. 1990.

${ }^{23}$ LA ROCHE, D. P.; CONNOLLY, D. A. L. Effects of stretching on passive muscle tension and response to eccentric exercise. American Journal of Sports Medicine, v. 34, n. 6, p. 1000-1007, june 2006.

${ }^{24}$ GLEIM G.W.; McHUGH M.P. Flexibility and its effects on sports injury and performance. Sports Medicine, Auckland, v. 24, n. 5, p. 289-299, nov. 1997.

${ }^{25} \mathrm{McHUGH}, \mathrm{M}$. P. et al. The role of passive muscle stiffness in symptoms of exerciseinduced muscle damage. American Journal of Sports Medicine, Baltimore, v. 27, n. 5, p. 594-599, oct. 1999.

${ }^{26}$ ARMSTRONG, R. B.; WARREN, G. L.; WARREN, J. A. Mechanisms of exerciseinduced muscle fibre injury. Sports Medicine, Auckland, v.12, n. 3, p. 184-207, sept. 1991.

${ }^{27}$ SOARES, J. M. C. et al. Children are less susceptible to exercise-induced muscle damage than adults: a preliminary investigation. Pedriatric Exercise Science, Champaign v. 8, n. 4, p. 361-367, nov. 1996.

${ }^{28}$ MARGINSON, V. F.; ESTON, R. G. The relationship between torque and joint angle during knee extension in boys and men. Journal of Sports Sciences, Londres, v.19, n. 11, p. $875-880$, nov. 2001.

${ }^{29}$ ESTON, R. G. et al. Effect of flexibility training on symptoms of exercise-induced muscle damage: a preliminary study. Journal of Exercise Science and Fitness, Hong Kong, v. 5, n. 1, p. 33-39, sept. 2007.

${ }^{30}$ CLARKSON, P. M. et al. Serum creatine kinase activity following forearm flexion isometric exercise. European Jornal of Applied Physiology, Heidelberg, v. 53, n.4, p. 368-371, apr. 1985.

Conexões: revista da Faculdade de Educação Física da UNICAMP, Campinas, v. 12, n. 3, p. 79-105, jul./set. 2014. 
${ }^{31} \mathrm{KOH}$, T. J.; BROOKS, S. V. Lengthening contractions are not required to induce protection from contraction-induced muscle injury. American Journal of Physiology Regulatory Integrative and Comparative Physiology, Bethesda, v. 281, n. 1, p. 155161 , july 2001.

${ }^{32}$ CORDOVA, A.; NAVAS, F. J. Os radicais livres e o dano muscular produzido pelo exercício: papel dos antioxidantes. Revista Brasileira de Medicina do Esporte, São Paulo, v. 6, n. 5, out. 2000.

${ }^{33}$ MESQUITA, I. C. Lesão muscular induzida por bupivacaínaem linhagens de camundongos predispostos a perfil distinto de citocinas. 2007. 101 f. Dissertação (Mestrado em Neuroimunologia) - Instituto de Biologia, Universidade Federal Fluminense, Niterói, Rio de Janeiro, 2007.

${ }^{34}$ CHEN, T. C.; NOSAKA, K.; SACCO, P. Intensity of eccentric exercise, shift of optimum angle and the magnitude of repeated bout effect. Journal of Applied Physiology, Bethesda, v. 102, n. 3, p. 992-999, nov. 2007.

${ }^{35} \mathrm{CHEN}, \mathrm{T}$. C. et al. Muscle damage responses of the elbow flexors to four maximal eccentric exercise bouts performed every 4 weeks. European Journal of Applied Physiology, Heidelberg, v. 106, n. 2, p. 267-275, may 2009.

${ }^{36} \mathrm{CHEN}, \mathrm{T}$. C. Effects of a second bout of maximal eccentric exercise on muscle damage and electromyographic activity. European Journal of Applied Physiology, Heidelberg, v. 89, n. 2, p. 115-121, apr. 2003.

${ }^{37}$ CHEN, T. C.; NOSAKA, K. Responses of elbow flexors to two strenuous eccentric exercise bouts separated by three days. Journal of Strength and Carditioning Research, Colorado Springs, v. 20, n. 1, p. 108-116, feb. 2006.

${ }^{38}$ PHILIPPOU, A. et al. Changes in the angle-force curve of human elbow flexors following eccentric and isometric exercise. European Journal of Applied Physiology, Heidelberg, v. 93, n. 1-2, p. 237-244, oct. 2004. 
${ }^{39}$ RODENBURG, J.B. et al. Warm-up, stretching and massage diminish harmful effects of eccentric exercise. International Journal of Sport Medicine, Stuttgart, v.15, p.414-419, oct. 1994.

${ }^{40}$ SAFRAN, M. R.; SEABER, A. V.; GARRETT, W. E. J. R. Warm-up and muscle injury prevention: an update. Sports Medicine, Auckland, v. 8, p. 239-249, oct. 1989.

${ }^{41}$ MCNAIR, P.; STANLEY, S. Effect os passive stretching and jogging on the series muscle stiffness and range of motion of the ankle joint. British Journal of Sports Medicine, Loughborough, v. 30, p. 313-318, dec. 1996.

${ }^{42}$ SHELLOCK, F. G.; PRENTICE, W. E. Warming-up and stretching for improved physical performance and prevention of sports-related injuries. Sports Medicine, Auckland, v. 2, n. 4, p. 267-278, july 1985.

${ }^{43}$ NOSAKA, K.; CLARKSON, P. M. Influence of previous concentric exercise on eccentric exercise-induced muscle damage. Journal of Sports Science, Londres, v.15, p. 477-483, oct. 1997.

${ }^{44}$ MAGNUSSON, S. et al. Passive energy absortion by human muscle- tendon unit is unaffected by increase in intramuscular temperature. Journal of Applied Physiology, Bethesda, v. 88, p. 1215-1220, apr. 2000.

${ }^{45}$ EVANS, R.K. et al. Effects of warm-up before eccentric exercise on indirect markers of muscle damage. Medicine and Science in Sports and Exercise, Hagerstown, v. 34, p. 1892-1899, dec. 2002.

${ }^{46} \mathrm{KOH}, \mathrm{T}$. J. Do small heat shock proteins protect skeletal muscle from injury? Exercise and Sport Science Review, Hagerstown, v.30, n. 3, p.117-121, july 2002.

${ }^{47}$ OISHI, Y. et al. Muscle type-speciffic responses of HSP60, HSP72, and HSC73 during recovery after elevation of muscle temperature. Journal of Applied Physiology, Bethesda, v. 92, p.1097-1103, mar. 2002.

Conexões: revista da Faculdade de Educação Física da UNICAMP, Campinas, v. 12, n. 3, p. 79-105, jul./set. 2014. 
${ }^{48}$ SELSBY, J. T.; DODD, S. L. Heat treatment reduces oxidative stress and protects muscle mass during immobilization. American Journal of Physiology Regulatony Integrative and Comparative Physiology, Bethesda, v. 289, p. R134-R139, july 2005.

${ }^{49}$ MCARDLE, A. et al. Overexpression of HSP70 in mouse skeletal muscle protects against muscle damage and age-related muscle dysfunction. FASEB Journal, Bethesda, v.18, p. 355-357, feb. 2004.

${ }^{50}$ MYIABARA, E. H. et al. Overexpression of inducible $70-\mathrm{kDa}$ heat shock protein in mouse attenuates skeletal muscle damage induced by cryolesioning. American Journal of Physiology, Baltimore, v. 290, n. 4, p. 1128-1138, apr. 2006.

Recebido em: 27 jun. 2014

Aceito em: 24 set. 2014

Contato: leonardocrlima@gmail.com 